\title{
a-Functionalization of Ketones via a Nitrogen Directed Oxidative Umpolung
}

Gabriel M. Kief ${ }^{[a]}$ and Tanja Gulder ${ }^{*[a, b]}$

In memory of Prof. Kilian Muñiz

\author{
[a] G. M. Kiefl, Prof. Dr. T. Gulder \\ Department of Chemistry, Technical University Munich, \\ Lichtenbergstrasse 4, 85748 Garching, Germany \\ [b] Prof. Dr. T. Gulder \\ Institute of Organic Chemistry, Leipzig University \\ Johannisallee 29, 04103 Leipzig, Germany \\ E-mail: tanja.gulder@uni-leipzig.de \\ Supporting information for this article is given via a link at the end of the document.
}

Abstract: Reversing the polarity in molecules is a versatile tool for expanding the boundaries of structural space. Despite a manifold of different umpolung methods available today, overcoming the inherent reactivity still remains a constant challenge in organic chemistry. The oxidative $\alpha$-functionalization of ketones by external nucleophiles constitute such an example. Herein, we present a hypervalent $\mathrm{F}$-iodane mediated umpolung of pyridyl ketones triggered by Lewis base Lewis acid non-covalent interactions. A wide variety of external nucleophiles are introduced with high regioselectivity applying this substratedirecting concept.

The a-functionalization of ketones 1 builds a corner stone in organic synthesis with electrochemical, ${ }^{[1]}$ photoredox ${ }^{[2]}$ and organocatalytic $^{[3]}$ approaches leading to important recent advances. The intrinsic polarization of ketones 1 , however, offer a nucleophilic (blue, Scheme 1) center at the a-position that determines the inherent reactivity and thus limits the addressable transformations of reactions employing electrophiles (red, Scheme 1a). The Umpolung concept (Scheme 1b), originally coined by Seebach and Corey for reversing the reactivity at acyl $C$ atoms, ${ }^{[4]}$ has led to a tremendous expansion of the reaction scope entering new structural territory not accessible with conventional methods. Reversing the polarity of carbonyl compounds ${ }^{[5]}$ can be accomplished with the help of various mediators, such as simple halogens, ${ }^{\left[{ }^{[6]}\right.}$ hypervalent iodanes, ${ }^{[7]}$ Lewis acids $^{[8]}$ and transition-metal catalysts. ${ }^{\left[{ }^{[9]}\right.}$

The first hypervalent iodane triggered Umpolung of ketones 1 was described already in 1978 by Mizukami. ${ }^{[10]}$ Applying this concept, a multitude of several functional groups ${ }^{[11]}$ have been transferred ranging from acetoxylations $\mathbf{s}^{[10,119]}$ to azidations $^{[11 f]}$ and halogenations ${ }^{[11 e]}$ until today. However, when using ketones $\mathbf{1}$, the nucleophile must be bound directly to the hypervalent iodane reagent. This drawback is associated with an enormous synthetic effort and restricts the scope of this method to the transfer of a few selected nucleophiles that are able to form chemically stable and isolable $\lambda^{3}$-hypervalent iodane reagents 2 . The preformation of the enol species as a silyl enol ether or Ir enolate $\mathbf{3}$ proved to be an elegant solution to this limitation and opened the door for applying external nucleophiles if linear iodane reagents $\mathbf{4}$ are used. $\alpha$-Aminations, ${ }^{[12]}$ cyanations, ${ }^{[13]}$ azidations, ${ }^{[14]}$ (hetero)arylation, ${ }^{[15]}$ and cyclopropanation ${ }^{[16]}$ of pre-modified ketones have thus become available (scheme 1b). Despite this tremendous progress, specific enol/enolate formation, no matter whether in-situ or beforehand, constitutes still a limiting factor, as regiocontrol is difficult to achieve if more than one enolisable $C$ atoms are present. ${ }^{[11 \mathrm{~g}]}$ The Martin-Matute group recently reported on an elegant attempt to overcome this issue by generating

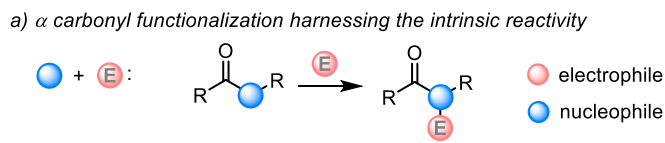

b) classical $\alpha$ carbonyl functionalization of simple ketones or enol ethers via inversion of polarity using $\lambda^{3}$-hypervalent iodane reagents

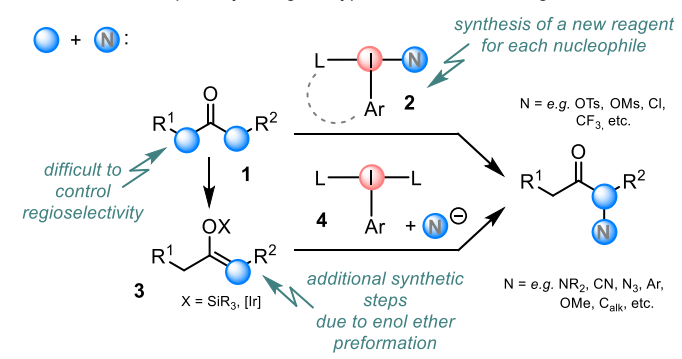

c) concept of this work: regioselective $\alpha$ carbonyl functionalizations via substrate-directed umpolung no enol ether

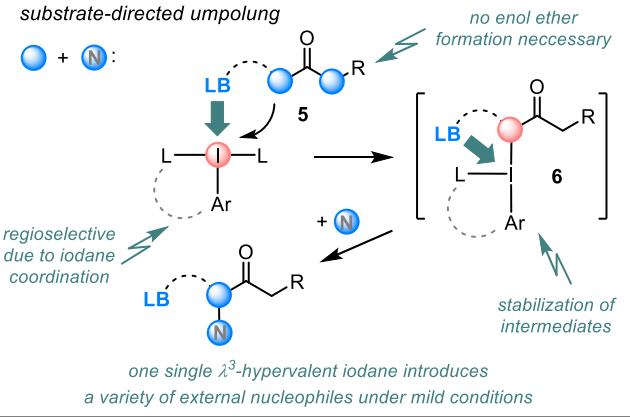

d) examples of inter- and intramolecular non-covalent interactions of Lewis basic groups and $\lambda^{3}$-hypervalent iodane reagents

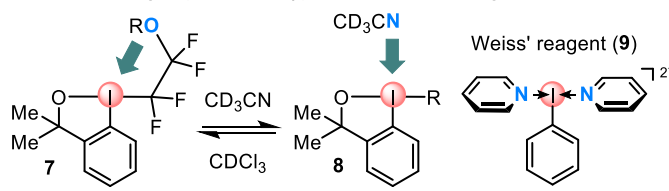

Scheme 1. General concepts for the oxidative a functionalization of carbonyls utilizing a) the intrinsic and b) the reversed ketone polarity; c) our approach via substrate-directed umpolung of reactivity; d) literature examples of electrostatic interactions in iodanes with nucleophilic moieties. ${ }^{[19,20]}$

an Ir-enolate via isomerization of the corresponding ally alcohol. ${ }^{[17]}$

Given our general interest in $\lambda^{3}$-hypervalent $F$-iodane mediated reactions together with the use of fluorine atoms as traceless directing groups to trigger unusal reactivity, ${ }^{[18]}$ we were wondering if the problem of regioisomerism in the a-functionalization of ketones can be overcome by precoordination of the hypervalent iodane reagent to a specific site of the substrate. The substrate-directed, now intramolecular nucleophilic addition of the enol to the 
electrophilic iodane furnishes regioselectively intermediate 6 which exhibits a reversed polarity compared to $\mathbf{5}$. Displacement of the hypervalent iodane nucleofug by an external nucleophile yields the target compound in a proximity driven reaction/intermediate stabilization event. In particular fluorine containing hypervalent iodanes, such as 16 and 17, show significant secondary bonding between the iodine atom and intra- $\mathbf{7}$ and intermolecular nucleophiles $\mathbf{8}$ (Scheme 1d). ${ }^{[19]}$ Inspired by the preliminary work of the Well's group in $1994,{ }^{[20]}$ we chose pyridines as the Lewisbasic, directing element in our investigations. Its established coordinating characteristics with hypervalent iodane atoms is impressively exemplified by the Weiss reagent (9). ${ }^{[21]}$ In addition, the pyridyl ring is among the most important structural motifs in bioactive compounds, ${ }^{[22]}$ making the exploration of new functionalization pathways highly desirable.

We set out to explore our substrate-directing $\lambda^{3}$ hypervalent-iodane mediated Umpolung of pyridyl ketones 10 that is devoid of the need for enol preformation and allows the application of external nucleophiles. To test the feasibility of our proposed concept, o-pyridyl 2-propanone (10a) together with benzotriazole $(\mathbf{1 1}, \mathrm{BTA}-\mathrm{H})$ as the external nucleophile were chosen as standard substrates.

Table 1. Optimization of the reaction conditions.

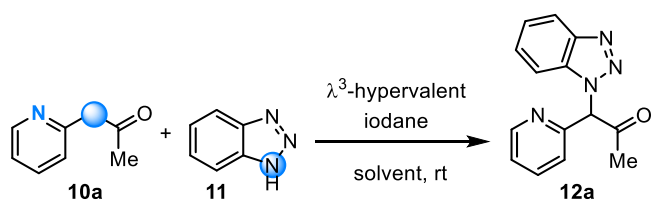

\begin{tabular}{|c|c|c|c|}
\hline entry & iodane & solvent & yield $6 \mathbf{a}^{[c]}$ \\
\hline 1 & $13^{[\mathrm{a}]}$ & DCM & $<5 \%$ \\
\hline 2 & $14^{[\mathrm{a}]}$ & $\mathrm{DCM}$ & $<5 \%$ \\
\hline 3 & $15^{[\mathrm{a}]}$ & DCM & $25 \%[d]$ \\
\hline 4 & $16^{[\mathrm{a}]}$ & DCM & $53 \%$ \\
\hline 5 & $17^{[\mathrm{a}]}$ & DCM & $55 \%$ \\
\hline 6 & $17^{[b]}$ & DCM & $72 \% / 66 \%[d]$ \\
\hline 7 & $17^{[a]}$ & MeCN & $<5 \%$ \\
\hline
\end{tabular}

a] 10a $(0.1 \mathrm{mmol})$, iodane $(0.11 \mathrm{mmol})$ and BTA-H $(\mathbf{1 1}, 0.11 \mathrm{mmol})$ were stirred for $1 \mathrm{~h}$ in a $0.2 \mathrm{M}$ solution ${ }^{[\mathrm{b}]} \mathbf{1 0 a}(0.11 \mathrm{mmol}), 17(0.10$ $\mathrm{mmol})$ and BTA-H $(\mathbf{1 1}, 0.30 \mathrm{mmol})$ were stirred at $\mathrm{rt}$ in DCM $(0.2$ $M)$. ${ }^{[c]}$ yield determined from the ${ }^{1} \mathrm{H}-\mathrm{NMR}$ spectrum using an internal standard. ${ }^{[d]}$ isolated yield.

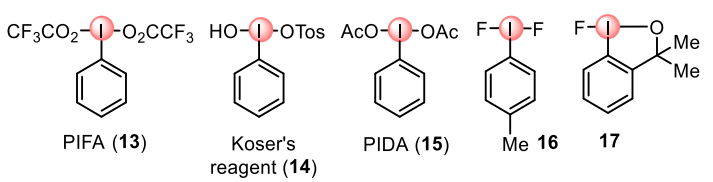

The model reaction was performed in the non-coordinating solvent DCM in order to avoid competitive interactions. Screening of several $\lambda^{3}$-hypervalent iodane reagents 13 - 17 (Table 1) disclosed both the linear 16 and the cyclic Fiodane $1^{[23]}$ as superior. Product $12 \mathrm{a}$ was formed as a single regioisomer in $53 \%$ and $55 \%$ yield, respectively with small amounts of $\mathrm{N}, \mathrm{N}$-disubstituted product present. The reagents 13 - 15 which have previously been the gold standard in such Umpolung reactions failed completely. PIFA (13) and Koser's reagent (14) led to an instantaneous decomposition even at lower temperatures while the less oxidative PIDA (15) gave a 1:2-mixture of the target compound $12 \mathrm{a}$ and the corresponding acetoxy derivative $\mathbf{1 2 g}$. Since $\mathbf{1 7}$ is, in general, easier to handle than 16, all further optimizations were carried out with the cyclic $F$ benzoiodoxole 17. Variations of the reaction conditions, such as changing the temperature or the solvent, did not improve the reaction outcome (cf. SI). Adjusting the ratio of $17(0.91 \mathrm{eq})$ and $11(3 \mathrm{eq})$ abolished the formation of $\mathrm{N}, \mathrm{N}$ disubstituted side product and thus increased the yield to $72 \%(66 \%$ isolated yield).

It is noteworthy that the use of MeCN (Table 1, entry 7), which has been the optimum medium in all fluoro functionalizations employing $\mathbf{1 7}$ hitherto reported by our group, ${ }^{[18]}$ failed completely. This supports our hypothesis of a non-covalent interaction between the pyridyl ketone 10a and the electron-deficient iodine atom in 17 being decisive for a successful conversion. With MeCN another, yet competitive Lewis basic compound was added in huge quantities to the reaction mixture and thus significantly inhibits pyridine-17 coordination by forming an MeCN-17 complex instead. A similar behavior has been described by Togni and Lüthi for other F-benziodoxole compounds (see 8 in Scheme 1d). ${ }^{[19]}$ Our mechanistic proposal was further verified by positioning the interacting nucleophilic entity at the meta $10 \mathrm{~b}$ or para position $10 \mathrm{c}$ of the pyridine ring or using the benzyl analog 18 devoid of the strong Lewis basic site (Scheme 2). In all cases no conversion was detected. Only the activated benzyl silyl enol ether $\mathbf{2 0}$ showed some turnover. Interestingly, here BAT-H (11) solely attacked with its $\mathrm{N}-2$ and not with the $\mathrm{N}-1$ atom as observed for all pyridyl ketones 10. The corresponding N-2 addition product 21 was isolated in $24 \%$ yield. In contrast, when using the o-pyridyl enol ether 22 no difference in the chemical yield of the obtained addition product 12a was detected (66\%). Overall, these preliminary mechanistic studies emphasize the importance of the presence and the position of the Lewis basic nitrogen atom ortho to the acidic $C-H$ functionality and thus corroborate its role as a directing (5, see Scheme 1c) and intermediate stabilizing group (6, Scheme 1c). A reaction proceeding via an in-situ formed pyridinium species such as $\mathbf{2 3}$ can be ruled out as treating ketone 10a solely with 17 delivered almost quantitatively untouched starting materials 10a and 17.

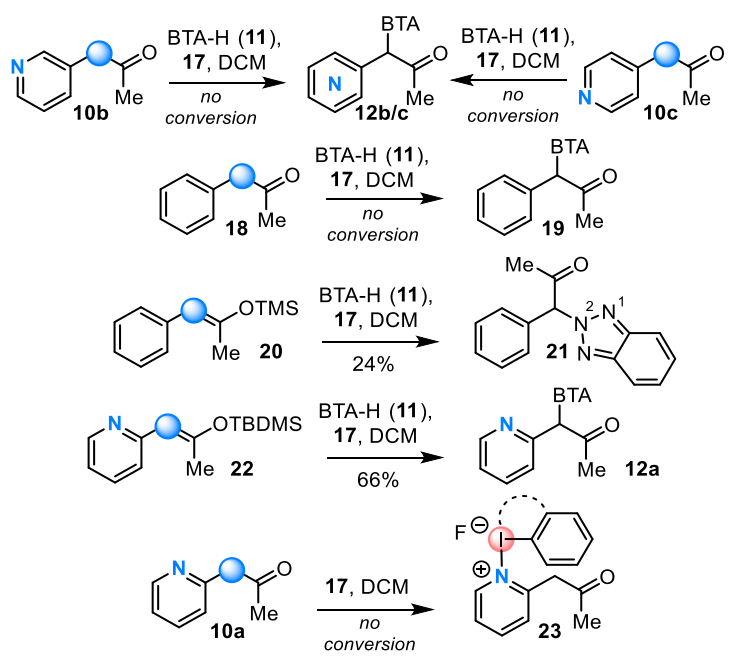

Scheme 2. Preliminary studies on the role of the pyridyl nitrogen.

Next, we turned our attention to the versatility of our developed procedure. The method showed a remarkably broad variety in the external nucleophile (Scheme 3). Besides different $N$-nucleophiles, such as heterocycles $(\rightarrow$ $\mathbf{1 2 d}$ and 12e) and sulfonamides ( $\rightarrow \mathbf{1 2 f}$ ), a wide range of structurally different $O$ - and $S$-nucleophiles were selectively installed giving the products $12 \mathrm{~g}-\mathbf{1 2} \mathrm{r}$ in good to excellent 
$30 \%-85 \%$ yields. The mildness of the method accounted for the tolerance of multiple, even oxidation sensitive functional groups such as alkenes, alkynes, furans, carbamates and phosphates. The method can also be extended to structurally more complex and/or chiral structures as showcased by the amino acid alanine $(\rightarrow 12 \mathrm{I})$ and santonic acid $(\rightarrow \mathbf{1 2 m})$. No racemization of the existing stereogenic centers occurred and even extra acidic $\mathrm{C}-\mathrm{H}$ groups were well accepted.

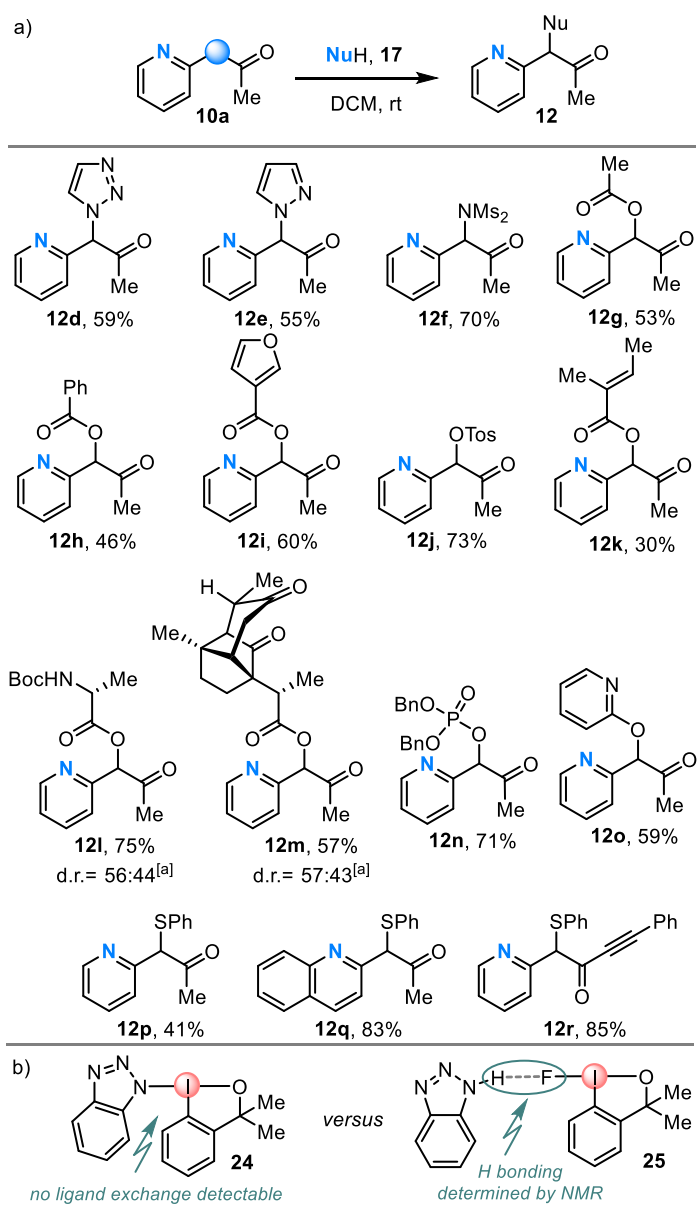

10a $(0.11 \mathrm{mmol}), 17(0.10 \mathrm{mmol})$ and $\mathrm{NuH}(0.30 \mathrm{mmol})$ were stirred in DCM $(0.2 \mathrm{M}) .{ }^{[\mathrm{a}]} \mathrm{d} . \mathrm{r}$. was determined from the ${ }^{1} \mathrm{H}$-NMR spectrum of the crude mixture.

Scheme 3. a) $\alpha$ Functionalizations of pyridyl ketone 10a with varying external nucleophiles and b) ligand exchange forming 24 versus $\mathrm{H}$ bonding interactions of $\mathbf{1 1}$ and $\mathbf{1 7 .}$

To our surprise, we found out that only nucleophiles exhibiting an acidic proton were successfully implemented in this transformation (for failed nucleophiles see SI). NMR spectra obtained from the titration of iodane $\mathbf{1 7}$ with increasing amounts of BTA-H (11) showed a significant upfield shift of the $\mathrm{N}-1$ proton signal in $\mathbf{1 1}$ and simultaneously a downfield shift of the $F$ resonance in $\mathbf{1 7}$ (cf. SI for spectra). Both observations provide evidence for an $\mathrm{H}$-bonding interaction like $\mathbf{2 5}$ which activates the BTA-H nucleophile (11) and at the same time the iodine(III) atom in 17. Basic aqueous work-up of these mixtures led to the recovery of both substrates $\mathbf{1 1}$ and $\mathbf{1 7}$ in quantitative yields thus excluding a likewise possible umpolung of the BTA moiety by generating a BTA-benziodoxole 24 in situ via ligand exchange (Scheme $3 b$ ).
Thorough analysis of the crude reaction mixture revealed that small amounts $(5-14 \%)$ of $\alpha$ fluorinated side products were built if carboxylic acids served as external nucleophiles (cf. in Scheme 3).

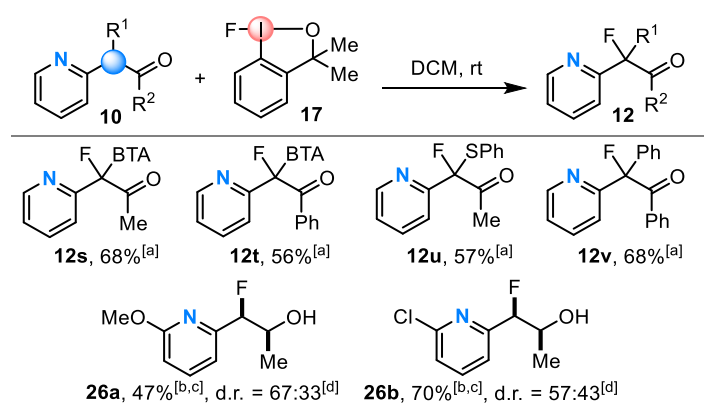

[a] 10 (0.20 mmol), $17(0.20 \mathrm{mmol})$ and pyridine HF $(0.60 \mathrm{mmol})$ were stirred in dry DCM $(0.2 \mathrm{M})$ in a Teflon vial. ${ }^{[\mathrm{b}]} \mathbf{1 0}(0.20 \mathrm{mmol})$ and $\mathbf{1 7}$ $(0.20 \mathrm{mmol})$ were stirred in DCM $(0.2 \mathrm{M})$. After completion $1.00 \mathrm{~mL}$ $\mathrm{MeOH}$ and $\mathrm{NaBH}_{4}(1.00 \mathrm{mmol})$ were added. ${ }^{[c]}$ yield over 2 steps. ${ }^{[d]}$ d.r. was determined from the ${ }^{1} \mathrm{H}-\mathrm{NMR}$ spectrum of the crude mixture.

Scheme 4. a Fluorinations of pyridyl ketones 10.

Since fluorinated compounds are, in general, extremely valuable substances whose applications range from pharmacy, ${ }^{[24]}$ medical diagnostics, ${ }^{[25]}$ agrochemistry ${ }^{[26]}$ to materials science, ${ }^{[27]}$ we thought changing our protocol to yield selectively the fluorinated ketones 12 would be worthwhile. Addition of pyridine HF to increase the concentration of fluoride and at the same time activate the $\mathrm{F}$-iodane 17 via $\mathrm{H}$-bonding gave the optimum conditions for forming the F-ketones. Their isolation, however, was impossible due to decomposition if an acidic proton was present in $\alpha$-position. ${ }^{[28]}$ Changing the substrate to $\alpha$ disubstituted ketones 10s - 10v solved this problem and furnished the target compounds $12 \mathrm{~s}-12 \mathrm{v}$ in $56 \%-68 \%$. Equipping the pyridine with electron-donating groups, like e.g., $\mathrm{Cl}$ or $\mathrm{OMe}$, and thus accelerating the $\mathrm{N}$-Lewis basicity, proved to be an excellent approach, too. The synthesis of the desired fluorinated products, which were isolated as the alcohols $\mathbf{2 6 a}$ and $\mathbf{2 6 b}$ due to the chemical instability of the corresponding ketones in $47 \%$ and $70 \%$ yield (over two steps) was accomplished even without additional HF.

The method also turned out to be highly flexible upon structural variations at the ketone $\mathbf{1 0}$. In all cases, oxidative functionalization of $\mathbf{1 0}$ proceeded regio- and chemoselectively with up to $81 \%$ yield (Scheme 5), preconditional a $\mathrm{N}$ atom in ortho position. Besides other $\mathrm{N}$ heterocycles, different substituents at the pyridine were equally well accepted. Here again, the reaction clearly benefited from an increase of the electron-density at the pyridine nitrogen evidenced by a significant rate acceleration. The range of tolerated substituents at the other site of the carbonyl $\left(R^{2}\right)$ span from alkyl, aryl, and even benzyl portions to alkynyl and electron-withdrawing fragments, such as $\mathrm{CF}_{3}$ or esters. The enamide 10al was successfully converted as well to the product 12 al in $51 \%$ yield indicating that carbonyl surrogates are likewise applicable. 


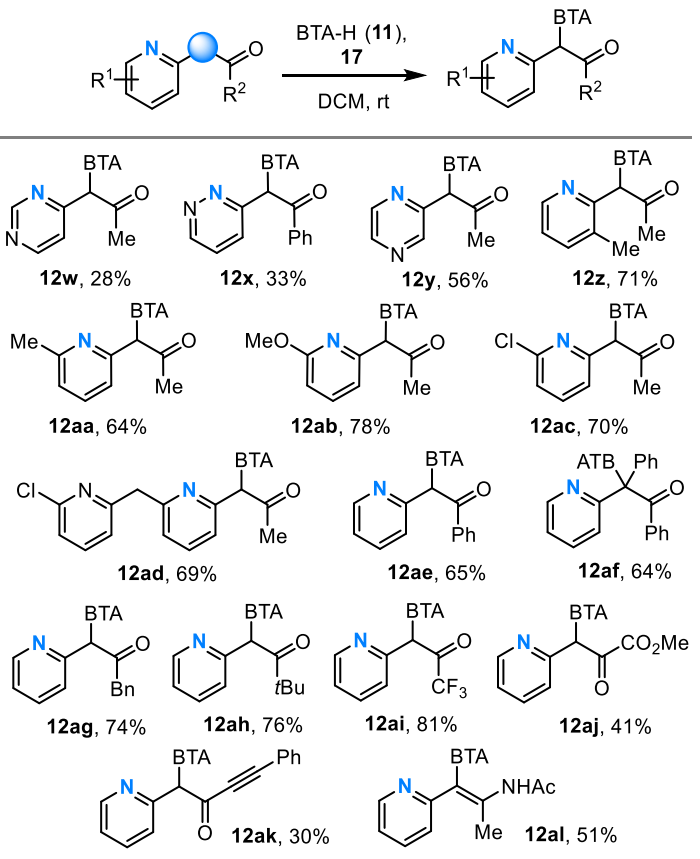

$10(0.11 \mathrm{mmol}), 17(0.10 \mathrm{mmol})$ and $11(0.30 \mathrm{mmol})$ were stirred in $\operatorname{DCM}(0.2 \mathrm{M})$.

Scheme 5. Oxidative a functionalizations of various ketones $\mathbf{1 2}$

In summary, we established an as yet unprecedented and efficient synthetic strategy for the oxidative umpolung of ketones 10. To realize our goal, we combined the unusual reactivity triggered by hypervalent $F$-iodanes ${ }^{[29]}$ and the directing, activating and stabilizing properties of the pyridine moiety. The addition of nucleophiles to the a-position of ketones 10 only takes place with 2-pyridyl substrates, in the presence of the hypervalent fluoro iodane 17. Preliminary mechanistic investigations unambiguously showed that the pyridine ring in $\mathbf{1 0}$ and the $\lambda^{3}$-fluoro iodane $\mathbf{1 7}$ are causally related with the observed transformation and this relationship can, most likely, be attributed to a non-covalent electrostatic interaction. The power of this concept was demonstrated by the synthesis of over 30 novel $\alpha$ functionalized ketones $\mathbf{1 2}$ in a straightforward manner with no relevant background reactions observable. Further investigations on the underlying complex principles operative here and the extension of this concept to other reaction types are currently underway in our laboratory.

\section{Acknowledgements}

This work was funded by the Emmy-Noether (GU 1134/3) and Heisenberg program (GU 1134/4) of the DFG.

Keywords: Umpolung - Hypervalent lodane • Substrate-directing Functionalization

[1] S. Liang, K. Xu, C.-C. Zeng, H.-Y. Tian, B.-G. Sun, Adv. Synth. Catal. 2018, 360, 4266-4292.

[2] Y. Liu, W. Dong, Chin. J. Chem. 2017, 35, 1491-1500.

[3] a) G. Guillena, in Comprehensive Enantioselective Organocatalysis (Ed.: P. I. Dalko), 2013, pp. 757-790; b) H.E. Lee, D. Kim, A. You, M. H. Park, M. Kim, C. Kim, Catalysts 2020, 10, 861

[4] a) D. Seebach, E. J. Corey, J. Org. Chem. 1975, 40, 231-237; b) D. Seebach, Angew. Chem. Int. Ed. 1979, 18, 239-258.

[5] O. Miyata, T. Miyoshi, M. Ueda, Arkivoc 2013, 2, 60-81.

[6] A. W. Erian, S. M. Sherif, H. M. Gaber, Molecules 2003, 8, 793-865.
[7] a) E. Merritt, B. Olofsson, Synthesis 2011, 4, 517-538; b) I. F. D. Hyatt, L. Dave, N. David, K. Kaur, M. Medard, C. Mowdawalla, Org. Biomol. Chem. 2019, 17, 7822-7848; c) D. Q. Dong, S.-H. Hao, Z.-L. Wang, C. Chen, Org. Biomol. Chem. 2014, 12, 4278-4289; d) A. Yoshimura, V. V. Zhdankin, Chem. Rev. 2016, 116, 3328-3435; e) V. V. Zhdankin, P. J. Stang, Chem. Rev. 2008, 108, 5299-5358; f) D. P. Hari, P. Caramenti, J. Waser, Acc. Chem. Res. 2018, 51, 3212-3225

[8] a) Y.-K. Wu, C. R. Dunbar, R. McDonald, M. J. Ferguson, F. G. West, J. Am. Chem. Soc. 2014, 136, 14903-14911; b) T. Miyoshi, T. Miyakawa, M. Ueda, O. Miyata, Angew. Chem. Int. Ed. 2011, 50, 928-931.

[9] Z. Xu, H. Chen, Z. Wang, A. Ying, L. Zhang, J. Am. Chem. Soc. 2016, 138, 5515-5518.

[10] F. Mizukami, M. Ando, T. Tanaka, J. Imamura, Bull. Chem. Soc. Jpn. 1978, 51, 335-336.

[11] a) J. S. Lodaya, G. F. Koser, J. Org. Chem. 1988, 53, 210212; b) G. F. Koser, J. S. Lodaya, D. G. Ray, P. B. Kokil, J. Am. Chem. Soc. 1988, 110, 2987-2988; c) G. F. Koser, A. G. Relenyi, A. N. Kalos, L. Rebrovic, R. H. Wettach, J. Org. Chem. 1982, 47, 2487-2489; d) R. M. Moriarty, H. Hu, S. C. Gupta, Tetrahedron Lett. 1981, 22, 1283-1286; e) G. C. Geary, E. G. Hope, K. Singh, A. M. Stuart, RSC Adv. 2015 5, 16501-16506; f) J. C. Lee, S. Kim, W. C. Shin, Synth Commun. 2000, 30, 4271-4275; g) M. Ochiai, Y. Takeuchi, T. Katayama, T. Sueda, K. Miyamoto, J. Am. Chem. Soc. 2005, 127, 12244-12245; h) I. Kieltsch, P. Eisenberger, A. Togni, Angew. Chem. Int. Ed. 2007, 46, 754-757.

[12] P. Mizar, T. Wirth, Angew. Chem. Int. Ed. 2014, 53, 59935997.

[13] H. Shen, J. Li, Q. Liu, J. Pan, R. Huang, Y. Xiong, J. Org. Chem. 2015, 80, 7212-7218.

[14] M. V. Vita, J. Waser, Org. Lett. 2013, 15, 3246-3249.

[15] a) A. A. More, G. K. Pathe, K. N. Parida, S. Maksymenko, Y. B. Lipisa, A. M. Szpilman, J. Org. Chem. 2018, 83, 24422447 ; b) J. Li, A. Bauer, G. D. Mauro, N. Maulide, Angew. Chem. Int. Ed. 2019, 58, 9816-9819; c) S. Maksymenko, K. N. Parida, G. K. Pathe, A. A. More, Y. B. Lipisa, A. M. Szpilman, Org. Lett. 2017, 19, 6312-6315; d) S. Arava, J. N. Kumar, S. Maksymenko, M. A. Iron, K. N. Parida, P. Fristrup, A. M. Szpilman, Angew. Chem. Int. Ed. 2017, 56, 2599-2603.

[16] A. Bauer, G. Di Mauro, J. Li, N. Maulide, Angew. Chem. Int. Ed. 2020, 59, $18208-18212$

[17] A. Sanz-Marco, S. Martinez-Erro, M. Pauze, E. GómezBengoa, B. Martín-Matute, Nat. Commun 2019, 10, 5244.

[18] a) A. Andries-Ulmer, C. Brunner, J. Rehbein, T. Gulder, J. Am. Chem. Soc. 2018, 140, 13034-13041; b) C. Brunner, A. Andries-Ulmer, G. M. Kiefl, T. Gulder, Eur. J. Org. Chem. 2018, 2615-2621; c) A. Ulmer, C. Brunner, A. M. Arnold, A. Pöthig, T. Gulder, Chem. Eur. J. 2016, 22, 3660-3664.

[19] a) V. Matoušek, J. Václavík, P. Hájek, J. Charpentier, Z. E. Blastik, E. Pietrasiak, A. Budinská, A. Togni, P. Beier, Chem. Eur. J. 2016, 22, 417-424; b) H. Pinto de Magalhães, A. Togni, H. P. Lüthi, J. Org. Chem. 2017, 82, 11799-11805.

[20] I. P. Andrews, N. J. Lewis, A. McKillop, A. S. Wells, Heterocycles 1994, 43, 713-717.

[21] a) R. Weiss, J. Seubert, Angew. Chem. Int. Ed. Engl. 1994, 33, 891-893; b) R. Corbo, J. L. Dutton, Coord. Chem. Rev. 2018, 375, 69-79.

[22] E. Vitaku, D. T. Smith, J. T. Njardarson, J. Med. Chem. 2014 57, 10257-10274.

[23] a) C. Y. Legault, J. Prevost, Acta Crystallogr. 2012, 68, 1238; b) G. C. Geary, E. G. Hope, K. Singh, A. M. Stuart, Chem Commun. 2013, 49, 9263-9265; c) V. Matoušek, E. Pietrasiak, R. Schwenk, A. Togni, J. Org. Chem. 2013, 78, 6763-6768.

[24] a) E. P. Gillis, K. J. Eastman, M. D. Hill, D. J. Donnelly, N. A. Meanwell, J. Med Chem. 2015, 58, 8315-8359; b) J. Wang M. Sanchez-Rosello, J. L. Acena, C. del Pozo, A. E. Sorochinsky, S. Fustero, V. A. Soloshonok, H. Liu, Chem. Rev. 2014, 114, 2432-2506; c) S. Purser, P. R. Moore, S. Swallow, V. Gouverneur, Chem. Soc. Rev. 2008, 37, $320-$ 330 .

[25] a) M. Tredwell, V. Gouverneur, Angew. Chem., Int. Ed. 2012, 51, 11426-11437; b) P. W. Miller, N. J. Long, R. Vilar, A. D. Gee, Angew. Chem., Int. Ed. 2008, 47, 8998-9033.

[26] a) T. Fujiwara, D. O'Hagan, J. Fluorine Chem. 2014, 167, 1629; b) P. Jeschke, Pest Manage. Sci. 2010, 66, 10-27.

[27] R. Berger, G. Resnati, P. Metrangolo, E. Weber, J. Hulliger, Chem. Soc. Rev. 2011, 40, 3496-3508.

[28] P. López-Alvarado, C. Avendaño, J. C. Menéndez, Synthesis 1998, 1998, 186-194.

[29] S. V. Kohlhepp, T. Gulder, Chem. Soc. Rev. 2016, 45, 62706288. 